\title{
Phenotype and Genotype of Enterococcus faecalis Isolated from Root Canal and Saliva of Primary Endodontic Patients
}

\author{
Zaki Mubarak ${ }^{1}$ Widya Asmara², Tri Wibawa ${ }^{3}$, Boy Bachtiar ${ }^{4}$ \\ ${ }^{1}$ Faculty of Dentistry, Syiah Kuala University, Banda Aceh 23111, Indonesia \\ ${ }^{2}$ Department of Microbiology, Faculty of Veterinary Medicine, Gadjah Mada University, Yogyakarta 55281, Indonesia \\ ${ }^{3}$ Department of Microbiology, Faculty of Medicine, Gadjah Mada University, Yogyakarta 55281, Indonesia \\ ${ }^{4}$ Department of Oral Biology, Faculty of Dentistry, Universitas Indonesia, Jakarta 10430, Indonesia \\ Correspondence e-mail to: zakimubarak54@yahoo.com
}

\begin{abstract}
Objective: To investigate the phenotype and genotype of E. faecalis isolated from the root canal and saliva of primary endodontic patients with periapical lesions. Methods: Eighteen adult male and female individuals suffering from primary endodontic infection, either with or without periapical lesions, were involved in this study. Root canal scraping and saliva were collected from each subject and used for bacterial quantitation using a real-time polymerase chain reaction (RT-PCR). Enterococci were isolated using ChromAgar medium and then identified using both biochemical (Gram staining and catalase tests) and molecular biology (conventional PCR) methods. Gelatinase activity, polysaccharide capsul profile and mRNA ace expression level were determined using microbiological, biochemical and molecular biology approach, respectively. Genotype of E. faecalis was determined based on nucleotide sequence of ace and gelE genes analyzed using web-based 3730xl DNA Analyze software. Results: The results showed that except for its proportion, no significant difference was found in phenotypes (gelatinase activity and mRNA ace expression levels) and genotypes (polymorphism of Cps operon and variation of ace and gelE nucleotide sequences) of E. faecalis isolated from the root canal and saliva of primary endodontic patients had or had no periapical lesions. Conclusion: It can be concluded that E. faecalis proportion had a role in the occurrence of periapical lesions in the primary endodontic patients, but not gelatinase activity, mRNA ace expression level, Cps operon polymorphism or ace and gelE nucleotide sequence variations.
\end{abstract}

Key words: Ace, Cps, Enterococcus, GelE, sequence

\section{INTRODUCTION}

The commensal bacterium Enterococcus faecalis is a non-oral microbiota that may inhabit in mouth cavity. ${ }^{1,2}$ Once occur in oral cavity, infected pulp or root canal $E$. faecalis may enter the entire system of root canal and survive to induce a number of dental problems although chemomechanic treatments have been applied. ${ }^{3}$ As consequences, E. faecalis becomes the most common bacteria isolated from the mouth cavity of patients suffering from a number of primary or secondary (posttreatment) endodontic infections. ${ }^{4-11}$ Here, E. faecalis may occur as a single infection agent or a mix infection with $E$. faecalis as the most dominant species. ${ }^{12}$
High infectivity of E. faecalis in human and animal tissues or organs and its remarkable endogenous resistance to extreme environmental conditions and antimicrobials may relate to this bacterium's ability to produce some virulence factors such as hemolysin (Hln), aggregation substance (AS) and gelatinase (GelE) as well as adhesin for collagen from $E$. faecalis (Ace) and polysaccharide capsule (Cps). ${ }^{13-16}$ Evidences for the role of Hln, AS and GelE in the pathogenesis of E. faecalis infection, so far, come from in vivo inflammation studies in nonendodontic animal models and from in vitro adherence test using certain cell lines. ${ }^{17-20}$ Additionally, the avail- 
able data explaining the relationship between the polymorphism of $E$. faecalis $\mathrm{Cps}$ and root canal infections is still controversial. ${ }^{21-23}$ Ace is the solely virulence factor of E. faecalis that has been known its role in facilitating the adherence of this bacterium in the collagen of human dentin. ${ }^{20}$ The data suggest that, except for that of Ace, the actual roles of virulence factors produced by $E$. faecalis in the pathogenesis of enterococcal infections in the root canal or dental tubuli are not clearly understood or experimentally proved.

Phenotype and genotype of E. faecalis could be characterized using a combination of microbiological and biochemical approaches supported by information obtained from molecular biology techniques such as conventional and real time polymerase chain reaction (RT-PCR) and DNA sequencing analysis. ${ }^{19,24}$ Since unique microenvironment may exist in certain regions of the mouth cavity, in particular between the root canal and saliva, it is assumed that there are some differences in the phenotype and genotype characteristic of virulence factors produced by E. faecalis lives in these two environments. $^{25}$

Unfortunately, there is no literature, so far, comprehensively describes the properties of virulence factors produced by E. faecalis lives in the root canal and saliva or explains the relationships between $E$. faecalis virulence factor genotype and phenotype diversities with the occurrence of periapical lesions in the primary endodontic patients. The objective of this study, therefore, was to investigate the relationship between phenotype (GelE activity and mRNA ace expression level) and genotype (polymorphism of Cps operons and ace and GelE nucleotide sequences) of $E$. faecalis isolated from the root canal and saliva with the incidence of periapical lesions in the patients suffered from primary endodontic infection.

\section{METHODS}

This study was approved by the Ethics Committee of Dentistry Faculty of Syiah Kuala University (Document no. 020/KG/FKG/2014). Subjects were 18 male and female individuals, aged 18-50 years old, who suffered from primary endodontic infection (4 with periapical lesions and 14 without periapical lesions) and voluntarily participated in the study after completing the informed consent. The status of necrotic pulp and periapical lesions was determined by thermal and radiographic examinations, respectively.

Root canal sample scrapings and saliva were aseptically collected from each subject using reamer and sterile cotton bud, respectively. Samples were placed in sterile plastic containers, diluted in $\mathrm{NaCl} 0.9 \%$ (E-Merck), and used for quantitation of bacterial proportion using RT-PCR technique. ${ }^{24}$ For isolation, samples were cultured in the ChromAgar medium (Brilliance VRE, Oxoid, Basingstoke, UK) for $18-24$ hours at $37^{\circ} \mathrm{C}$ and observed by binocular Olympus microscopy using 100x Phase contrast. ${ }^{26}$ The pink colonies isolates were then identified using Gram staining and catalase test. The colonies were also regrowth in the brain heart infussion (BHI) medium (Acumedia) containing 10\% fetal bovine serum (FBS) (Acumedia) before finally being identified by conventional PCR using E. faecalis $16 \mathrm{~S}$ rRNA primers. ${ }^{24,27}$

Phenotypes of E. faecalis examined were: (1) GelE activity that was determined based on the diameter of halo produced after this bacterium growth in the tryptic soy agar (TSA) medium (BD Diagnostic Systems) containing 1.5\% skim milk (Himedia Laboratories), and (2) and mRNA ace expression level that was quantitated by RT-PCR using $E$. faecalis ace forward and reverse primers. ${ }^{24}$ Genotype of the E. faecalis isolated was determined based on nucleotide sequences of ace and gelE genes analyzed using web-based 3730xl DNA Analyze software. Data obtained was tabulated and then analyzed descriptively.

\section{RESULTS}

\section{Isolation and identification of $\boldsymbol{E}$. faecalis}

Culturing root canal sample scrapings and saliva from primary endodontic patients with or without periapical lesions in the ChromAgar medium successfully recovered 38 Enterococcus isolates. Enterococcus spp., including E. faecalis and E. faecium, resulted in pink colonies in ChromAgar because they produced $\beta$-glucosidase that was able to change chromogenic substances in the medium. ${ }^{26}$

To differentiate $E$. faecalis (Gram positive and catalase negative) from $E$. faecium (Gram positive and catalase positive), the pink colonies obtained were screened further using Gram staining and catalase test, two biochemical methods commonly used to identify bacteria isolated from oral samples. ${ }^{9}$ Results of these biochemical tests showed that there were 19 E. faecalis from 38 Enterococcus colonies isolated using ChromAgar medium.

Proportion of $E$ faecalis based on the source of isolate The detection of E. faecalis isolates' DNA with specific primers that were subsequently amplified by quantitated PCR technique may explain the differences in the number and proportion of these bacteria according to the source of clinical samples used in this study (Figure 1). 


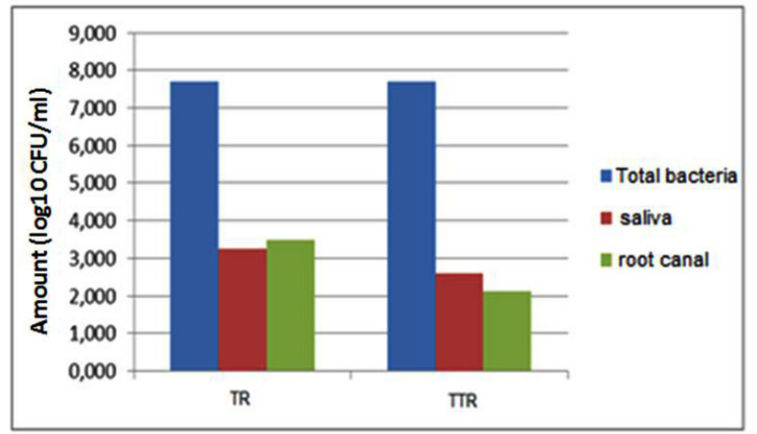

Figure 1. The number of E. faecalis based on the sources of sample (root canal and saliva) of primary endodontic patients had (TR) or had no (TTR) periapical lesions. Total DNA of E. faecalis and total bacteria was amplified using E. faecalis $16 \mathrm{~S}$ rRNA primers and universal primers, respectively. ${ }^{24,27}$

The results quantitated PCR analysis suggested that the higher numbers of $E$. faecalis in root canal (3.489 $\mathrm{CFU} / \mathrm{mL}$ ) than that in saliva $(3.236 \mathrm{CFU} / \mathrm{mL})$ of primary endodontic patients with periapical lesions.

\section{Gelatinase activity}

Using microbiology method described previously, GelE activity of E. faecalis isolated from the root canal and saliva of primary endodontic patients with or without periapical lesion was successfully determined as illustrated in Figure 2. ${ }^{24,26}$ Based on GelE activity showed by the control E. faecalis strain ATCC 29212, GelE activity was categorized into negative, weak $(0.1-0.2 \mathrm{~cm})$, medium $(0.3-0.8 \mathrm{~cm})$ or strong $(1.0-1.9 \mathrm{~cm})$.

The majority (84.2\%) of E. faecalis had GelE activity ranged from weak to strong, and only small amount $(15.8 \%)$ showed no GelE activity. In primary endodontic patients with periapical lesions there was no significant difference $(p>0.05)$ in the numbers of E. faecalis isolates live in root canal and saliva showed either weak or strong GelE activity. In primary endodontic patients without periapical lesions, E. faecalis isolates had a weak GelE activity were more prevalent in saliva (36.8\%) than in root canals, whereas strong GelE activity was produced by $E$. faecalis mainly isolated from root canals. However, there was no significant difference $(p>0.05)$ found in the activity of GelE E. faecalis isolates between the two groups of primary endodontic patients evaluated. This study also observed the incongruent GelE activity showed by $E$. faecalis isolated from root canal and saliva of one primary endodontic patient without periapical lesions.

\section{Level of mRNA ace expression}

Phenotype of Ace of E. faecalis isolated in this study was determined based on the level of mRNA

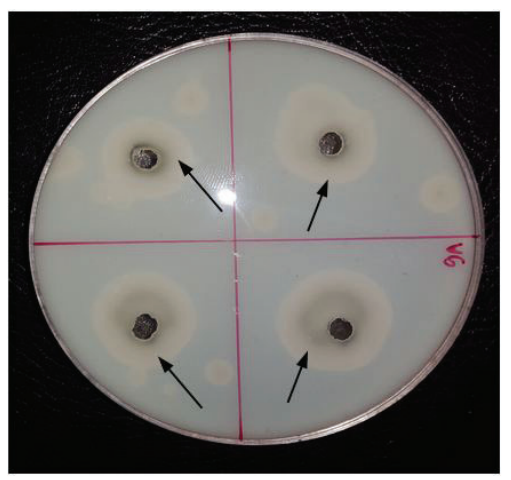

Figure 2. Gelatinase activity showed by $E$. faecalis isolates. Black arrows represent the halo of wells containing isolates cultures for 24 hour in the TSA medium.

ace expression quantitated using qPCR technique after the bacterium was cultured in the BHI solution containing $10 \%$ FBS. The results indicated that 8 out of 19 E. faecalis isolated from root canals and saliva of primary endodontic patients without periapical lesions expressed mRNA ace. According to the ratio, mRNA ace expression levels belonged into 3 categories: weak $(-0.87--2.63)$, moderate $(0.19-0.30)$, and strong (3.58 - 8.27). Here, weak mRNA ace expression level showed by the control E. faecalis ATCC 29212 was used a reference.

Isolates had moderate and strong mRNA ace expression levels showed higher $(p<0.05)$ mRNA ace expression compared to E. faecalis ATCC 29212. E. faecalis isolates had strong (4.47) mRNA ace expression were mainly found in primary endodontic patients had no periapical lesions whereas those having weak (0.08) mRNA ace expression were prevalent in primary endodontic patients with periapical lesions.

\section{Polymorphism of polysaccharide capsule}

Using the method described previously, this study successfully identified three types of Cps operons in E. faecalis lived in root canals and saliva of primary endodontic patients without periapical lesions, namely Cps-1 (73.7\%), Cps-2 (10.5\%) and Cps 5 (10.5\%). Only $5.3 \%$ (1/19) were Cps negative. ${ }^{40}$ The number of isolates had Cps 1, 2 and 5 found in the root canals or saliva of these primary endodontic patients slightly varied (Figure 3). The differences, however, were not statistically analyzed due to the limited number of samples and $E$. faecalis had Cps 2 and 5 operons.

Enterococcus faecalis isolates had Cps-1 operon, those did not produce polysaccharide capsule, were mainly isolated from root canals and saliva of primary endodontic patients without periapical lesions (47.3\%) 


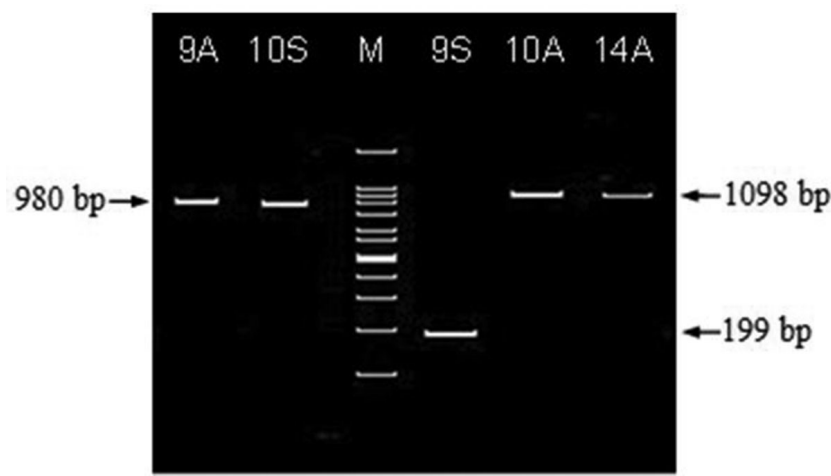

Figure 3. Electrogram showing the band of Cps1 (980 bp), Cps2 (1098 bp) and Cps 5 (199 bp) operons. Number stands for sample number. $\mathrm{M}=$ Marker, $\mathrm{A}=$ root canal, $\mathrm{S}=$ Saliva

whereas isolates had Cps-2 or Cps-5 operons were more prevalent in primary endodontic patients with periapical lesions. The dominance of non-Cps E. faecalis in the saliva of primary endodontic patients with periapical lesions indicated that saliva was a convenient environment for these strains compared to root canals. Further study is needed to do to explain this assumption.

\section{DISCUSSION}

Isolation and identification of $\boldsymbol{E}$. faecalis

Results of the biochemical tests showed that there were 19 E. faecalis from 38 Enterococcus colonies isolated using ChromAgar medium. Since detection of E. faecalis from oral samples required more specific and sensitive approaches such as a PCR that was able to amplify DNA encoding 16S rRNA, bacterial identification was also performed by PCR method using E. faecalis $16 \mathrm{~S}$ RNA primers. ${ }^{24,27-29}$ Similar to those of biochemical methods, this molecular biological approach confirmed the occurrence of 19 E. faecalis isolates from 38 Enterococci colonies recovered in the ChromAgar medium. It can be concluded that the occurrence of Enterococcus bacteria in ChromAgar medium cultured with root canal sample scrapings and saliva collected from primary endodontic patients did not necessarily indicate the presence of $E$. faecalis in the clinical samples analyzed.

Results of this study suggested that in the primary endodontic patients without periapical lesions, E. faecalis was more frequently found in saliva rather than in root canals. In the primary endodontic patients with periapical lesions, in contrast, equal numbers of E. faecalis were found in both root canal and saliva. These data indicated that saliva became the main source of $E$. faecalis in the mouth of patients from where clinical samples for this study were obtained. This finding was in agreement with results of previous studies reported that $E$. faecalis was consistently isolated from both root canal and saliva of patients suffered from primary and secondary endodontic infections. ${ }^{10,12,30-33}$ Data of this study, however, could not explain whether the isolated E. faecalis was part of mouth flora or just a transient bacterium in this organ as this organism could be present in the mouth cavity from dietary sources..$^{34-35}$

Proportion of $E$ faecalis based on the source of isolate The results quantitated PCR analysis suggested that the higher numbers of $E$. faecalis in root canal (3.489 CFU/ $\mathrm{mL})$ than that in saliva $(3.236 \mathrm{CFU} / \mathrm{mL})$ of primary endodontic patients with periapical lesions. This is contrast to condition found in the primary endodontic patients without periapical lesions where the numbers of $E$. faecalis in root canal $(2.117 \mathrm{CFU} / \mathrm{mL})$ were lower than that in saliva $(2.608 \mathrm{CFU} / \mathrm{mL})$. Overall, the numbers of $E$. faecalis in the root canal and saliva of primary endodontic patients had periapical lesions $(58.7 \%)$ were higher than that of primary endodontic patients without periapical lesions $(41.3 \%)$. The qPCR technique was also able to show dominance of $E$. faecalis population over other bacteria species in both root canal and saliva of these patients. These results support the notion that the qPCR was a sensitive technique for detection and quantitation of bacterial number and proportion in clinical oral samples. ${ }^{24}$

It was revealed from this study that periapical lesions were mainly found in primary endodontic patients with high number and proportion of E. faecalis in both root canal and saliva. Periapical lesions, on the other hand, were absent in primary endodontic patients had lower number and proportion of E. faecalis in root canal or saliva. These facts suggested that the high presence of E. faecalis in root canal and saliva was strongly related to the case of periapical lesions in primary endodontic patients.

\section{Phenotypes of $E$. faecalis \\ Gelatinase activity}

This study also observed the incongruent GelE activity showed by E. faecalis isolated from root canal and saliva of one primary endodontic patient without periapical lesions. Here saliva isolate was GelE negative, but root canal isolate showed weak GelE activity. This was not uncommon phenomenon because previous showed that although gelE gene existed in the chromosome of all $E$. faecalis cells, this gene was not regularly expressed..$^{36,37}$ Previous studies also found that $E$. faecalis isolated from oral cavity generally expressed GelE, but activity of the enzyme in vitro was heterogenic. ${ }^{24,38}$

\section{Level of mRNA ace expression}

Results obtained in this study were different from previous reports suggesting that E. faecalis expressed higher levels of mRNA ace when cultured in the 


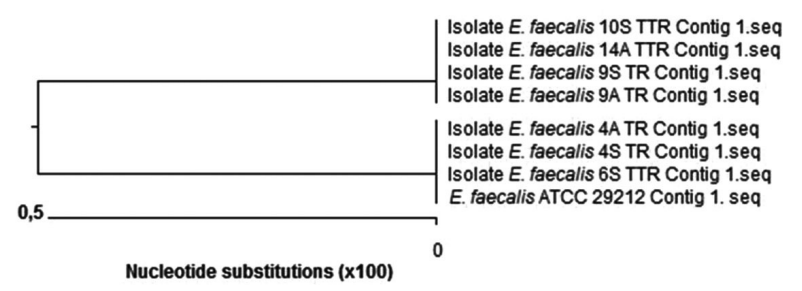

Figure 4. Phylogenic tree of $E$. faecalis isolates constructed based on gelE sequence analysis showing the difference and similarity of the isolates with overseas strains. $\mathrm{S}=$ saliva, $\mathrm{A}=$ root canal, $\mathrm{TR}=$ patients had periapical lesions; $\mathrm{TTR}=$ patients had no periapical lesions

serum E. faecalis or growth medium supplemented by serum. ${ }^{15,39}$ The mRNA ace expressions reported by these authors were not categorized as weak, moderate or strong as done in this study. Moreover, study did not address the relationship between mRNA ace expression level and the occurrence of periapical lesions. ${ }^{39}$

From this study could be assumed that level of mRNA ace expression in the E. faecalis live in the root canal was not directly related to the incidence of periapical lesions in primary endodontic patients. This was contrast to results showing that there was a positive correlation between the levels of mRNA ace expressed and $E$. faecalis infections in experimental endocarditis. More detailed research is, therefore, required to explain this assumption. ${ }^{15}$

\section{Genotypes of $E$. faecalis \\ Polymorphism of polysaccharide capsule}

Results obtained in this study were different from those reported. ${ }^{22}$ In their studies Bachtiar and coworkers reported that $E$. faecalis species had Cps-2 were more prevalent in primary and secondary endodontic patients. ${ }^{22}$ This difference might be caused by individual variation or unique environment triggering endodontic infections among populations. ${ }^{41,42}$ Similar to this study, Bachtiar and coworkers did not explain whether the $E$. faecalis had Cps 2 reported were isolates with positive or negative Cps phenotypes. According to Gaspar et al., E. faecalis had Cps 2 genotype consisted of 2 phenotypes, namely $\mathrm{Cps}$ positive and $\mathrm{Cps}$ negative. ${ }^{22,23}$ The two strains could be identified based on the occurrence of $6770 \mathrm{bp}$ insertion that could be detected using qPCR method. ${ }^{20}$ This study focused on Cps operons of E. faecalis in primary endodontic patients with or without periapical lesions, different from other researchers that did not address periapical lesions in the primary endodontic patients investigated..$^{22,23}$

\section{gelE and ace nucleotide sequences}

The variation observed in the activity of gelatinase and the level of mRNA ace expression of E. faecalis

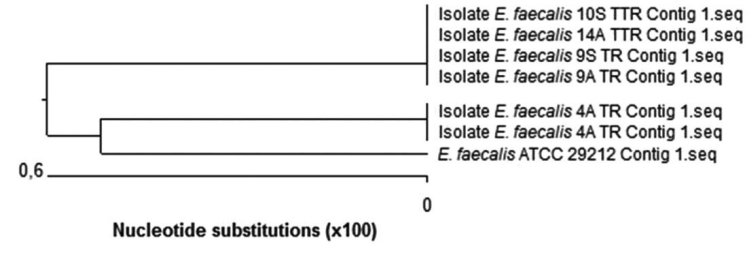

Figure 5. Phylogenic tree of E. faecalis isolates constructed based on ace sequence analysis showing the difference and similarity of the isolates with overseas strains. $\mathrm{S}=$ saliva, $\mathrm{A}=$ root canal, $\mathrm{TR}=$ patients had periapical lesions; $\mathrm{TTR}=$ patients had no periapical lesions

isolates collected from primary endodontic patients with or without periapical lesions might relate to the polymorphism of gelE and ace, the encoding genes. To address this assumption, coding regions for both gelE and ace preliminary were separately amplified by PCR, sequenced and then analyzed using web-based 3730xl DNA Analyze software programs. These unraveled similarity and difference of gelE and ace nucleotide sequences of $E$. faecalis isolates as shown by phylogenic tree of the isolates created according to the sequence of each gene (Figure 4 and 5).

Results of PCR amplification suggested that from $19 E$. faecalis isolates, 7 were gene gelE positive (Figure 4) and 6 were gene ace positive (Figure 5). Comparative analysis on the sequences of the two genes indicated that E. faecalis isolates found in the root canals of primary endodontic patients, either with or without periapical lesions, had $100 \%$ homology with those found in their saliva. It was predicted, therefore, that periapical lesions occurred in the patients were caused by E. faecalis strains in saliva entered root canals as reported by Mozini et al. and de Oliviera et al. ${ }^{43,44}$

Based on nucleotide sequences of ace and gelE, E. faecalis strains isolated from primary endodontic patients with or without periapical lesions involved in this study could be grouped into two clusters with 0.5-0.6 differences per 100 nucleotides. These small differences, however, were enough to classify the isolates into two separate branches in the phylogenic trees.

Similarity and difference of these E. faecalis isolated in this study with overseas isolates were also analyzed by comparing nucleotide sequences of gelE and ace of the isolates with those of overseas isolates downloaded from the database (http://www.ncbi.nlm.nih.gov). The results showed that the first cluster occurred in a branch separated from overseas isolates. There were four isolates in this cluster, namely $9 \mathrm{~A}, 9 \mathrm{~S}, 10 \mathrm{~S}$ and 14A. The first two isolates were obtained from the root canals and saliva of primary endodontic patients with 
periapical lesions, respectively; whereas the other two were obtained from saliva and root canals of primary endodontic patients without periapical lesions. These isolates (except for 10S), uniquely, showed very strong gelatinase activity.

Different from those in the first cluster, E. faecalis isolates in the second cluster showed closer relationships with a number of overseas isolates such as JH2-2 and collagen adhesin from America, 75A from India, TLME3 from China and 494-95 from Portugal as well as local isolates of South Korea, Japan and the control E. faecalis ATCC 29212. Two isolates in this cluster (4A and 4S) were collected from primary endodontic patients had periapical lesions, and one (6S) was collected from saliva of primary endodontic patient had no periapical lesions. These all three isolates showed weak gelatinase activity.

Nucleotide differences ranged from 0.5 upto 1.0 between $E$. faecalis strains isolated in this study and overseas E. faecalis isolates suggested specificity of these isolates. Contribution of this genetic specificity on the incidence of periapical lesions in primary endodontic patients, however, was not obviously identified. Further studies are, therefore, required to unravel the roles of gelE and ace polymorphisms on the incidence and pathogenesis of periapical lesions in primary endodontic patients.

\section{CONCLUSION}

Based on results of the study and discussion it can be concluded that high number of E. faecalis found in both root canal and saliva was related to the incidence of periapical lesions in primary endodontic patients. This is contrast to the insignificant relationship found between gelatinase activity, mRNA ace expression, Cps polymorphism, and genetic variation of gelE and ace with the occurrence of periapical lesions in these patients, respectively.

\section{ACKNOWLEDGMENT}

We would like to thank Dra. Dessy from the Faculty of Dentistry of Universitas Indonesia for her great help in laboratory analysis and for drh. Al Azhar, M.Kes, Ph.D from Faculty of Veterinary of Syiah Kuala University for his support and advice in the manuscript preparation.

\section{REFERENCES}

1. Aas JA, Paster JB, Stokes NL, Olsen I, Dewhirst EF. Defining the normal bacterial flora of the oral cavity. J Clin Microbiol. 2005; 43: 5721-32.

2. Wang QQ, Zhang FC, Chu CH, Zhu FX. Prevalence of Enterococcus faecalis in saliva and filled root canals of teeth associated with apical periodontitis. Int J Oral Sci. 2012;4:19-23.

3. Walton RE, Riviera ME, Torabinejad TM. Principles and Practice of Endodontic. Philadelphia: WB Saunders. 2002.

4. Kouidhi B, Zmantar T, Mahdouani K, Hentati H Bakhrouf A. Antibiotic resistance and adhesion properties of oral Enterococci associated to dental caries. BMC Microbiol. 2011;11: 155.

5. Rôças IN, Siqueira FJ, Santos RK. Association of Enterococcus faecalis with different forms of periradicular diseases. J Endod. 2003;30:315-20.

6. Souto R, Colombo PA. Prevalence of Enterococcus faecalis in subgingival biofilm and saliva of subjects with chronic periodontal infection. Arch Oral Biol. 2008;53:155-60.

7. Takahashi Y, Yoshida A, Nagayoshi M, Kitamura C, Nishihara T, et al. Enumeration of viable Enterococcus faecalis, a predominant apical periodontitis pathogen, using propidium monoazide and quantitative real-time polymerase chain reaction. Microbiol Immunol. 2011;55: 889-92.

8. Suchitra U, Kundabala M. Enterococcus faecalis: An endodontic pathogen. India: Indian Endodontic Society. 2006.

9. Hancock HH, Sigurdsson A, Trope M, Moiseiwitsch J. Bacteria isolated after unsuccessful endodontic treatment in a North American population. Oral Surg Oral Med Oral Pathol Oral Radiol Endod. 2001;91:579-86.

10. Portenier I, Waltimo TMT, Haapasalo M. Enterococcus faecalis the root canal survivor and 'star' in post-treatment disease. Endod Topics. 2003;6:135-59.

11. Zhu X, Qang Q, Zhang C, Cheung PSG, Shen Y. Prevalence, phenotype, and genotype of Enterococcus faecalis isolated from saliva and root canals in patients with persistent apical periodontitis. J Endod. 2010;36:1950-5.

12. Libertin CR, Dumitru R, Stein SD. The hemolysin/ bacteriocin produced by enterococci is a marker of pathogenicity. Diagn Microbiol Infect Dis. 1992;15:115-20.

13. Peciuliene V, Reynaud H, Balciuniene I, Haapasalo M. Isolation of yeasts and enteric bacteria in rootfilled teeth with chronic apical periodontitis. Int Endod J. 2010;34:429-34. 
14. Ike Y, Hashimoto H, Clewell BD. High incidence of hemolysin production by Enterococcus (Streptococcus) faecalis strains associated with human parenteral infections. J Clin Microbiol. 1987;25: 1524-8.

15. Singh KV, Nallapareddy RS, Sillanpaa J, Murray BE. Importance of the collagen adhesin Ace in pathogenesis and protection against Enterococcus faecalis experimental endocarditis. PLOS Pathog. 2010;6: e1000716.

16. Hufnagel M, Hancock LE, Koch S, Theilacker C, Gilmore MS, et al. Serological and genetic diversity of capsular polysaccharides in Enterococcus faecalis. J Clin Microbiol. 2004;42: 2548-57.

17. Ike Y, Hashimoto H, Clewell DB. Hemolysin of Streptococcus faecalis subspecies zymogenes contributes to virulence in mice. Infect Immun. 1984;45:528-530

18. Kreft B, Marre R, Schramm U, Wirth R. Aggregation substance of Enterococcus faecalis mediates adhesion to cultured renal tubular cells. Infect Immun. 1992;60:25-30.

19. Coque MT, Patterson JE, Steckelberg JM, Murray BE. Incidence of hemolysin, gelatinase, and aggregation substance among enterococci isolated from patients with endocarditis and other infections and from feces of hospitalized and communitybased persons. J Infect Dis. 1995;171:1223-9.

20. Kowalsky WJ, Kasper LE, Hatton FJ, Murray EB, Nallapreddy RS, Gillespie JM. Enterococcus faecalis adhesin, Ace, mediates attachement to particulate dentin. J. Endod. 2006;32:634-7.

21. Pinheiro ET, Penas PP, Endo M, Gomes PB, Mayer PM.Capsule locus polymorphism among distinct lineages of Enterococcus faecalis isolated from canals of root-filled teeth with periapikal lesions. J Endod. 2012;38: 58-61.

22. Bachtiar EW, Bachtiar BM, Dewiyani S, Akbar SMS. Enterococcus faecalis with capsule polysaccharides type 2 and biofilm-forming capacity in Indonesians requiring endodontic treatment. J Investig Clin Dent. 2015;1-9.

23. Gaspar, BF, Montero N, Akary E, Teixeira N, Matos R, et al. Incongruence between the cps type 2 genotype and host-related phenotypes of an Enterococcus faecalis food isolate. Int J Food Microbiol. 2012;158: 120-5.

24. Sedgley CM, Molander A, Fllannagan ES, Nagel $\mathrm{CA}$, Appelbe KO, et al. Virulence, phenotype and genotype characteristic, of endodontic Enterococcus. Oral Mircobiol Immunol. 2005;20:10-9.

25. Marcotte H, Lavoie MC. Oral microbial ecology and the role of salivary immunoglobulin A. Microbiol Mol Biol Rev. 1998;62:71-109.

26. Perry JD, Morris KA, James AL, Oliver M, Gould FK. Evaluation of novel chromogenic substrates for the detection of bacterial b-glucosidase. J Appl Microbiol. 2007;102: 410-5.
27. Williams JM, Martin T, Daniel CJ, Diane SC. Detection and quantitation of Enterococcus faecalis by real-time PCR (qPCR), reverse transcription-PCR (RT-PCR), and cultivation during endodontic treatment. J Endod. 2006;35: 715-21

28. Malani PN, Thal L, Donabedian MS, RobinsonDunn B, Kauffman AC, et al. Molecular analysis of vancomycin-resistant Enterococcus faecalis from Michigan hospitals during a 10 year period. J Antimicrob Chemother. 2002;49: 841-3.

29. Harwood JV, Delahoya NC, Ulrich RM, Kramer MF, Whitlock JE, et al. Molecular confirmation of Enterococcus faecalis and E. faecium from clinical, faecal and environmental sources. Lett Appl Microbiol. 2004;38: 476-82.

30. Sundqvist G, Fidgor D. Life as an endodontic pathogen: Ecological difference between untreated and filled root canal. Endod Topics. 2003;6:3-28.

31. Stojanović N, Krunić J, Popović B, Stojičić S, Zivković S. Prevalence of Enterococcus faecalis and Porphyromonas gingivalis in infected root canals and their susceptibility to endodontic treatment procedures: a molecular study. Srp Arh Celok Lek. 2014;535-41

32. Mahmoudpour A, Rahimi S, Sina M, Soroush $\mathrm{HM}$, Shahi S, et al. Isolation and identification of Enterococcus faecalis from necrotic root canals using multiplex PCR. J Oral Sci. 2007;49: 221-7.

33. Molander A, Relt C, Dahlen G, Kvist T. Microbiologic status of root-filled teeth with apical periodontitis. Int Endod J. 1998;31: 1-7.

34. Poulsen LL, Bisgaard M, Son NT, Trung NV, An HM, Dalsgaard A. Enterococcus faecalis clones in poultry and in humans with urinary tract infections in Vietnam. Emerging Infect Dis. 2012;18: 1096-100

35. Riboldi PG, Frazzon J, d'Azevedo PA, Frazzon APG. Antimicrobial resistance profile of enterococcus spp isolated from food In Southern Brazil. Brazilian J Microbiol. 2009;40:125-8.

36. Comerlato C, de Resende MCC, Caierão J, d'Azevedo PA. Presence of virulence factors in Enterococcus faecalis and Enterococcus faecium susceptible and resistant to vancomycin. Mem Inst Oswaldo Cruz. 2013;108:590-5.

37. Marra A, Dib-Hajj F, Lamb L, Kaczmarek F, Shang $\mathrm{W}$, et al. Enterococcal virulence determinants may be involved in resistance to clinical therapy. Diagn. Microbiol Infect Dis. 2007;58: 59-65.

38. Creti R, Imperi M, Lucia LB, Fabretti F, Orefici $\mathrm{G}$ et al. Survey for virulence determinant among Enterococcus faecalis isolated from different sources. J Med Microbiol. 2004;53:13-20.

39. Shepard DB, Gilmore MS. Differential expression of virulence-related genes in Enterococcus faecalis in response to biological cues in serum and urine. Infect Immun. 2002;70:4344-52. 
40. McBride MS, Fischetti VA, LeBlanc DJ, Moellering RC, Gilmore MS. Genetic diversity among Enterococcus faecalis. PLoS ONE. 2007;2: e582.

41. Kinane DF, Hart DF. Genes and gene polymorphisms associated with periodontal disease. Crit Rev Oral Biol Med. 2003;14:430-49.

42. Morsani MJ, Aminoshariae A, Han YW, Montagnese TA, Mickel A. Genetic Predisposition to Persistent Apical Periodontitis. J Endod. 2011;37:1-11.
43. Mozini ACA, Vansan LP, Neto MDS, Pietro R. Influence of the length of remaining root canal filling and post space preparation on the coronal leakage of Enterococcus faecalis. Brazilian J Microbiol. 2009;40:174-9.

44. de Oliveira DGS, Gomes DJ, Costa MHN, de Sousa ER, Lund RG. Coronal microleakage endodontically treated with intracanal post exposed to fresh human saliva. J Appl Oral Sci. 2013;21:403-8.

(Received December 4, 2015; Accepted 13 April 2016) 